\title{
Not Between the Eyes!
}
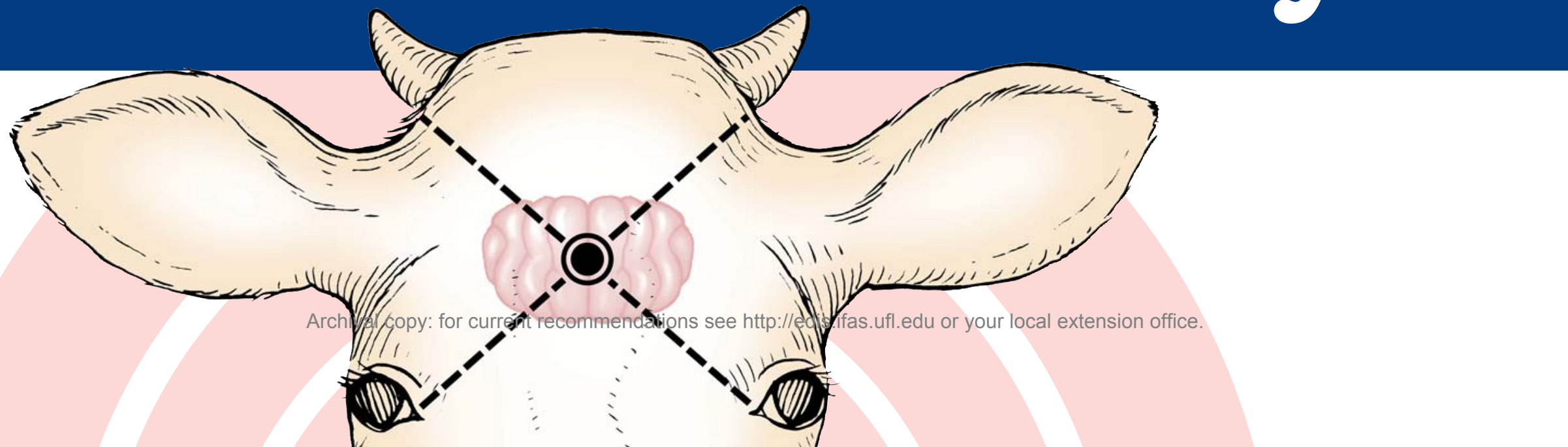

Point of Entry of projectile in cattle should be at the intersection of two imaginary lines, each drawn from the inside corner of the eye to the base of the opposite horn (or to a point slightly above the opposite ear in a cow without horns). Center of forehead, but NOT BETWEEN THE EYES!

In most circumstances on the farm or ranch, gunshot is the only practical method of euthanasia. This procedure requires the selection of an appropriate firearm and bullet with sufficient velocity, energy and size to pass through the skull (enter the brain), and cause massive brain destruction. A .22 caliber long rifle bullet fired from either a pistol or rifle is sufficient for young animals. Hollow or soft point .22 caliber bullets increase brain tissue destruction, but may not penetrate the skull in adult animals. Euthanasia of bulls, adult cows, horses, or Cervidae (elk) by gunshot requires larger calibers such as a $9 \mathrm{~mm}$ or .357 because of thickness of the skull. Proper placement of the bullet is essential and best achieved by holding the firearm within a few inches of the intended target. The firearm should not be held or placed against the head.
Gunshot Hold firearm perpendicular to the skull and within inches of the intended target when possible. A .22 caliber hollow or soft point bullet is sufficient for young animals. However, larger adult animals require at least a .22 magnum solid point bullet or preferably a $9 \mathrm{~mm}$ or .357 caliber bullet.

Penetrating Captive Bolt Restrain animal so that the device can be held firmly against the skull over the intended site. As described for gunshot, the penetrating captive bolt should be positioned perpendicular to the skull. Always insure death by exsanguination (bleeding out).

\section{Humane Euthanasia Procedures}

